\title{
Lipid Indices vs Anthropometric Indices in Metabolic Syndrome and Type 2 Diabetes Mellitus
}

\author{
Asha Augusthy ${ }^{1 *}$, Suchanda Sahu² and Ashok Kumar Jeppu ${ }^{3}$ \\ 'Dept of Biochemistry, Sree Narayana Institute of Medical Sciences, Ernakulam, Kerala, India \\ ${ }^{2}$ Department of Biochemistry, All India Institute of Medical Sciences, Sijua, Bhubaneshwar, Odisha, India \\ ${ }^{3}$ Biochemistry Unit, International, Medical School, Management and Science University, Shah Alam, Selangor, Malaysia
}

\section{ABSTRACT}

Background: Diabetes mellitus is a metabolic disease known by chronic hyperglycemia which results from defective insulin action and secretion. Metabolic Syndrome consists of a constellation of metabolic abnormalities that confer increased risk of diabetes mellitus. The aim of our study is to find out whether non-invasive, clinically measurable surrogates could be useful in identifying body fat distribution and help predict metabolic syndrome and diabetes risk and to compare the performance of anthropometric indices with lipid indices in identifying metabolic syndrome and diabetes.

Methods: 50 individuals with metabolic syndrome ,50 individuals with type 2 diabetes mellitus and 50 controls were selected by purposive sampling technique. For cases and controls history was taken, physical examination was done .Fasting blood sugar, Serum High density lipoprotein and Serum Triglyceride levels were estimated. Body mass index, a body shape index, visceral adiposity index, lipid accumulation factor was calculated.

Results: The mean values visceral adiposity index, lipid accumulation factor were significantly increased $(\mathrm{p}<0.001)$ in cases compared to controls.

Conclusion: Our study concluded that lipid indices visceral adiposity index, lipid accumulation factor is better than anthropometric indices like body mass index, a body shape index in predicting metabolic syndrome and type 2 diabetes mellitus. Anthropometric indices when used should be correlated with metabolic variables and clinical symptoms.

\section{Keywords: Metabolic Syndrome, Diabetes Mellitus, Body Mass Index, Body Shape Index, Visceral Adiposity Index, Lipid Accumulation Factor}

\section{Introduction}

Diabetes mellitus is a metabolic disease known by chronic hyperglycemia which results from defective insulin action and secretion. World Health Organization projects that number of diabetics will exceed 350 million by 2030 . Metabolic syndrome (MetS) consists of a constellation of metabolic abnormalities that confer increased risk of diabetes mellitus. The major features of the metabolic syndrome include central obesity, hypertriglyceridemia, low HDL (high density lipoprotein) cholesterol, hyperglycemia and hypertension ${ }^{[1]}$.

Body Mass Index (BMI) is one of the largely used screening tool in identification of metabolic syndrome. BMI fails to distinguish between body fat and muscle mass and thus has its drawbacks in predicting metabolic syndrome ${ }^{[2]}$. MRI and CT are now considered the gold standard for the quantitative evaluation of visceral adipose tissue and subcutaneous adipose tissue ${ }^{[3]}$.

VAI (Visceral Adiposity index) estimates visceral fat distribution and it is a useful determinant of the phenotype change and substituted the necessity to take high-cost imaging studies, thereby making the prediction much more practical in daily clinical practice and populations studies for the assessment of cardio metabolic risk associated with visceral obesity ${ }^{[4]}$. In addition, VAI showed a correlation with known adipocytokines and cardio metabolic risk serum markers ${ }^{[5-6]}$.

A body shape index (ABSI), based on normalizing waist circumference (WC) to BMI and height. The advantage of ABSI is that it combines information from WC, height and weight. A high ABSI indicates that $\mathrm{WC}$ is higher than expected for a given height and weight, and corresponds to a more central concentration of body mass ${ }^{[7]}$.

Lipid accumulation factor (LAP) combines waist measurements and fasting triglyceride (TG) levels, reflecting both the anatomic and physiological changes associated with lipid over accumulation. LAP was closely associated with cardiovascular diseases (CVD), diabetes and metabolic syndrome and outperformed BMI for identifying these diseases ${ }^{[8] .}$ 
The aim of our study is to find out whether non-invasive, clinically measurable surrogates could be useful in identifying body fat distribution and help predict metabolic syndrome and diabetes risk. We also want to examine the associations of anthropometric and lipid indices with metabolic syndrome and diabetes risk, and to compare the performance of anthropometric indices with lipid indices in identifying metabolic syndrome and diabetes.

\section{Materials and Methods}

Study design- The present study was conducted in the department of Biochemistry, Father Muller's medical college after obtaining clearance from institutional ethics committee. The study group consisted of 150 individuals selected by purposive sampling technique who had come to hospital for health check-up during a time period of two years. Informed written consent was obtained from all individual participants included in the study. This was a case-control study with a sample size of 150 patients.

Selection of subjects- 50 individuals with metabolic syndrome (all patients who fulfil criteria for metabolic syndrome, according to National cholesterol education program (NCEP): ATP III 2001 for metabolic syndrome $\left.{ }^{[9]}\right), 50$ individuals with type 2 diabetes mellitus and 50 controls (age above 40 years, 38 males and 12 females and not a diabetic or patient with metabolic syndrome).

Exclusion criteria- Smokers, alcoholics, patients with history of liver and renal impairment were excluded from the study.

Sample and data collection- For the selected patient's history was taken, physical examination was done. FBS,
Serum HDL and Serum Triglyceride levels were estimated. FBS was estimated using GOD-POD method. Triglycerides were estimated by enzymatic colour test GPO-PAP method. HDL was estimated by immune-inhibition enzymatic colour test. All estimations were done on Olympus AU 400 autoanalyzer.

BMI was calculated as weight $(\mathrm{kg})$ divided by square of the height $\left(\mathrm{m}^{2}\right)$. VAI score was calculated as described using the following sex-specific equations, $\mathrm{WC}$ is expressed in $\mathrm{cm}$, TG is expressed in $\mathrm{mmol} / \mathrm{L}$ and $\mathrm{HDL}$ levels expressed in $\mathrm{mmol} / \mathrm{L}$ [10]: Men: [WC/39.68+(1.88*BMI)]*(Triglycerides/1.03) *(1.31/HDL-C), Women: $[\mathrm{WC} / 36.58+(1.89 * \mathrm{BMI})] *($ Triglycerides $/ 0.81) *(1.52 /$ HDL-C).

ABSI was calculated as $\mathrm{WC}(\mathrm{m}) /\left(\mathrm{BMI}^{2 / 3} *\right.$ height $\left.(\mathrm{m})^{1 / 2}\right)$, expressed in $\mathrm{m}^{11 / 6} \mathrm{~kg}^{-2 / 3[11]}$. LAP was calculated using WC $(\mathrm{cm})$ and TG level $(\mathrm{mmol} / \mathrm{L})$ using the following formula for men and women respectively ${ }^{[12]} \mathrm{LAP}=(\mathrm{WC}-65) * \mathrm{TG}$ for men $\mathrm{LAP}=(\mathrm{WC}-58) * \mathrm{TG}$ for women

Statistical analysis- The data was analysed by ANOVA for multiple group comparisons and Pearson's correlation coefficient for relationship between variables. Statistical analyses were performed with the help of SPSS software. For all statistical analyses the $p$ value was considered to be significant when $\mathrm{p}<0.05$.

\section{Results}

There was a statistically significant difference between VAI $\left(\mathrm{F}_{(2.147)}=12.294, \mathrm{p}=.000\right)$ and $\operatorname{LAP}\left(\mathrm{F}_{(2.147)}=11.338\right.$, $\mathrm{p}=.001$ ) on each group (Control, Diabetic, Metabolic syndrome) using statistical test ANOVA.

Table 1: Correlation of diastolic blood pressure DBP), systolic blood pressure (SBP, Fasting blood sugar (FBS), High Density Lipoprotein (HDL), Triglyceride (TG), waist circumference (WC) with visceral Adiposity Index (VAI), A Body Shape Index (ABSI) and Lipid Accumulation factor (LAP) in controls, diabetics and metabolic syndrome.

\begin{tabular}{|c|c|c|c|c|c|c|c|}
\hline & & \multicolumn{2}{|l|}{ VAI } & \multicolumn{2}{|l|}{ ABSI } & \multicolumn{2}{|l|}{ LAP } \\
\hline & & r value & $p$ value & r value & $p$ value & r value & P value \\
\hline \multirow[t]{6}{*}{ Control } & Diastolic BP & -.097 & .504 & -.200 & .164 & -.062 & .668 \\
\hline & Systolic BP & -.079 & .588 & -.200 & .163 & -.047 & .744 \\
\hline & FBS & -.217 & .131 & -.240 & .093 & -.172 & .231 \\
\hline & HDL & .080 & .579 & .016 & .914 & -.032 & .823 \\
\hline & TG & .850 & $.000^{* *}$ & $.759^{* *}$ & .000 & .490 & $.000^{* *}$ \\
\hline & Waist circumference & .110 & .447 & .105 & .467 & .027 & .853 \\
\hline \multirow[t]{6}{*}{ Diabetic } & Diastolic BP & .067 & .643 & .165 & .252 & .367 & $.009^{* *}$ \\
\hline & Systolic BP & .367 & $.009^{* *}$ & .252 & .078 & .439 & $.001^{* *}$ \\
\hline & FBS & .127 & .380 & .173 & .230 & .176 & .222 \\
\hline & HDL & -.460 & $.001^{* *}$ & .113 & .433 & -.135 & .349 \\
\hline & TG & .797 & $.000^{* *}$ & -.267 & .061 & .926 & $.000^{* *}$ \\
\hline & Waist circumference & .071 & .623 & .277 & .052 & .453 & $.001^{* *}$ \\
\hline
\end{tabular}




\begin{tabular}{|c|c|c|c|c|c|c|c|}
\hline & & \multicolumn{2}{|l|}{ VAl } & \multicolumn{2}{|l|}{ ABSI } & \multicolumn{2}{|l|}{ LAP } \\
\hline & & r value & $p$ value & r value & $p$ value & r value & $P$ value \\
\hline \multirow[t]{6}{*}{ Metabolic syndrome } & Diastolic BP & .091 & .531 & .162 & .261 & .141 & .328 \\
\hline & Systolic BP & .030 & .838 & .294 & $.038^{*}$ & .134 & .352 \\
\hline & FBS & -.171 & .234 & -.239 & .095 & -.110 & .445 \\
\hline & HDL & -.369 & $.008^{* *}$ & .168 & .243 & -.193 & .180 \\
\hline & TG & .948 & $.000^{* *}$ & -.172 & .233 & .948 & $.000^{* *}$ \\
\hline & Waist circumference & .285 & $.045^{*}$ & .519 & $.000^{* *}$ & .431 & $.002^{* *}$ \\
\hline
\end{tabular}

** Correlation is significant at the 0.01 level (2-tailed). * Correlation is significant at the 0.05 level (2-tailed).

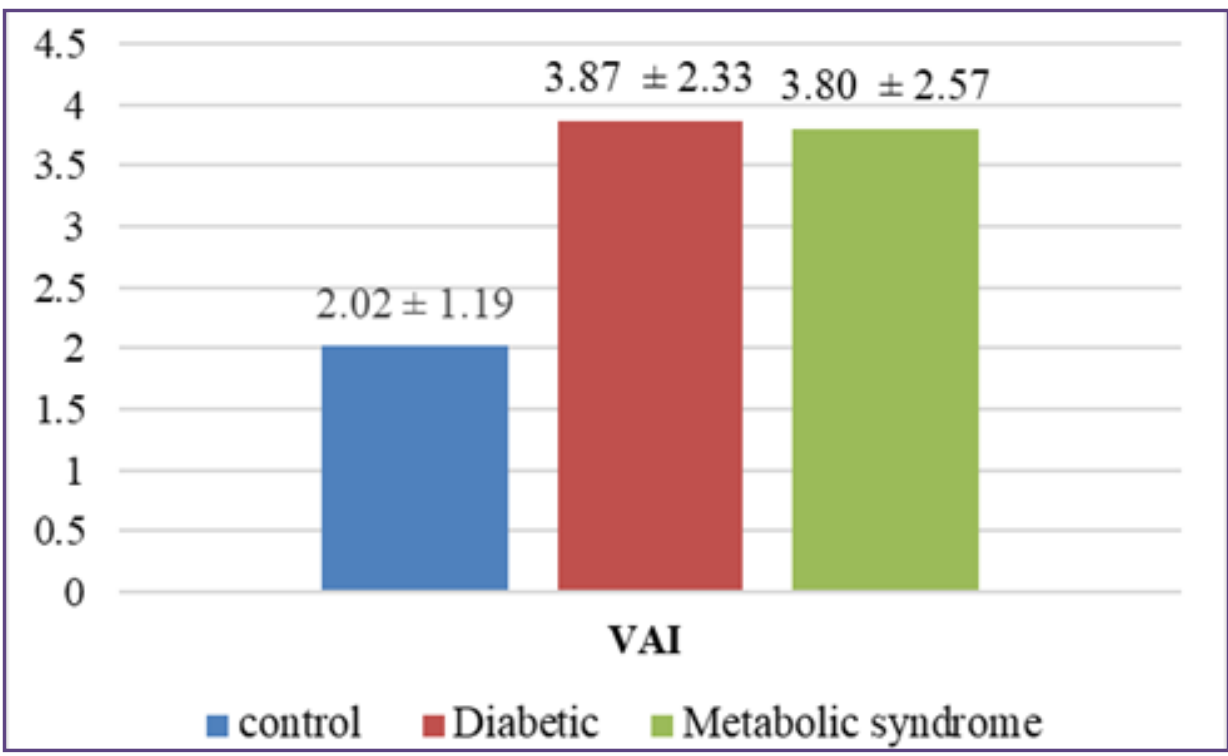

Fig. 1: Comparison of visceral adiposity index between control, diabetic and metabolic syndrome using ANOVA (F (2,147) $=12.294, p<0.001$ ).

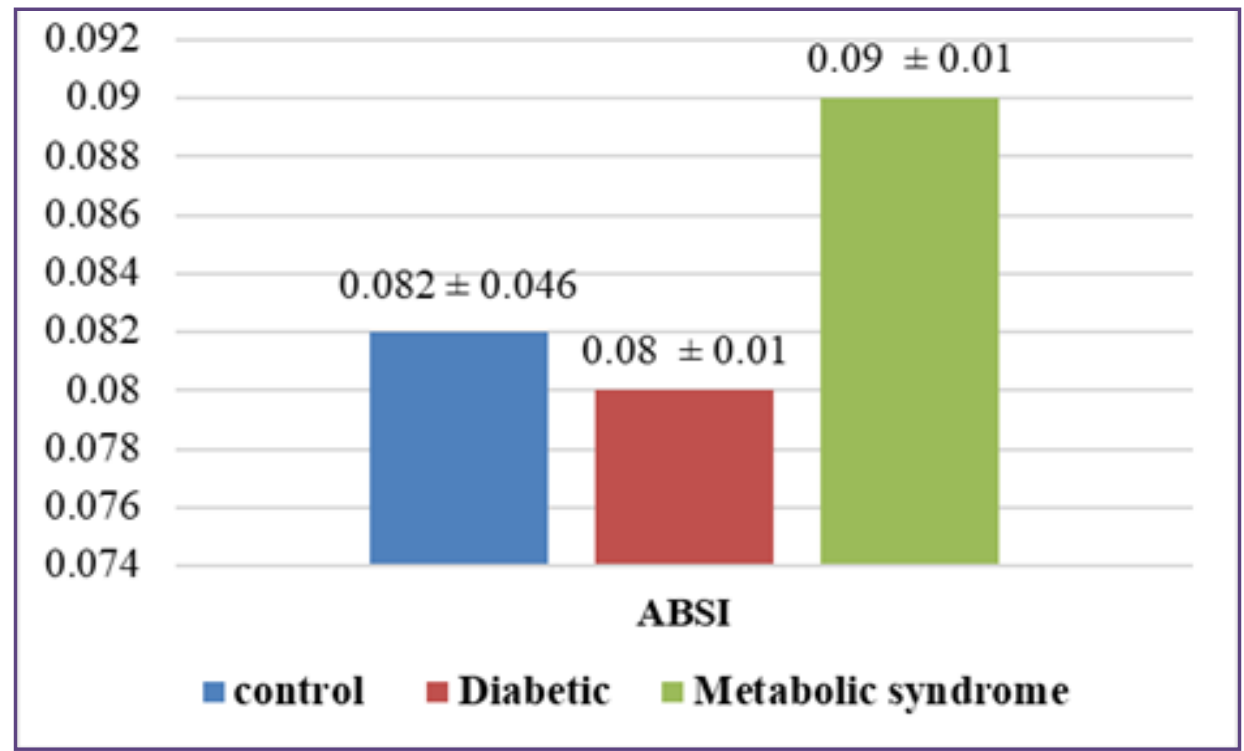

Fig. 2: Comparison of a body shape index between control, diabetic and metabolic syndrome $(p$ value $=0.082)$ using ANOVA. 


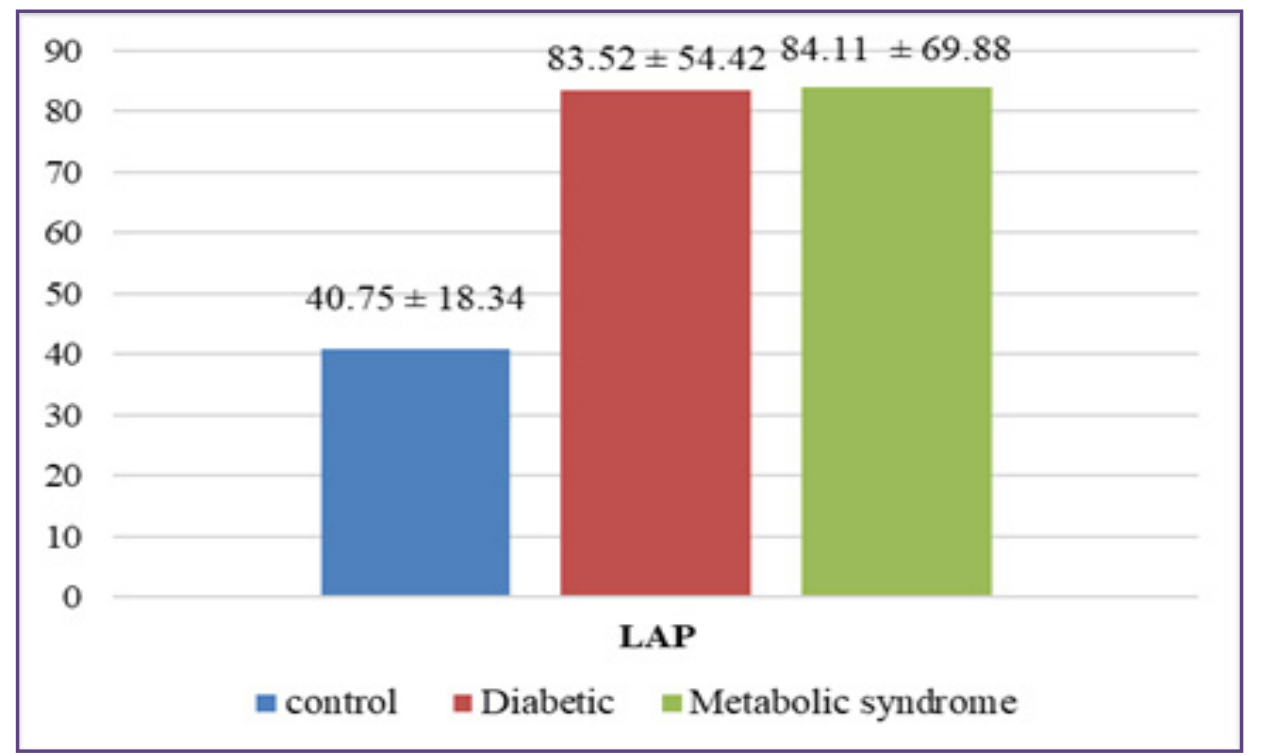

Fig. 3: Comparison of lipid accumulation factor between control, diabetic and metabolic syndrome using ANOVA (F (2,147) $=11.338, \mathrm{p}=\mathbf{0 . 0 0 1})$.

\section{Discussion}

Cardiometabolic risk (CMR) denotes a cluster of metabolic abnormalities predictive of cardiovascular diseases (CVD), which is 3-4 times identifiable among individuals with Type-2 Diabetes Mellitus (T2DM) ${ }^{[13]}$.Therefore it is important to use reliable markers that readily identify patients at risk of CVDs. This study analysed comparative usefulness of VAI, ABSI and LAP in identifying individuals at risk of CVDs.

VAI was found to be significantly increased in patients with metabolic syndrome and type 2 diabetes. A major risk factor for diabetes is visceral obesity ${ }^{[14]}$. VAI is suggested as a surrogate of visceral adipose and it includes both anthropometric and metabolic variables. VAI provides information regarding visceral adipose tissue and insulin resistance ${ }^{[15]}$. Visceral obesity which has been proposed as a marker of adiposity dysfunction and ectopic fat deposition is more metabolically deleterious than general obesity or subcutaneous fat and it leads to lipotoxicity and insulin resistance ${ }^{[16]}$. A prospective cohort study conducted in China showed that the VAI is a better surrogate index than single anthropometric indices ${ }^{[17]}$. Even though VAI cannot be considered as a diagnostic tool for cardiovascular events, as it includes physical and metabolic parameters, it will reflect other risk factors like altered production of adipocytokines, increase in lipolytic activity and plasmafree fatty acids. In patients with visceral obesity a state of relative hypoleptinemia can be observed in patients when compared with generalized obesity. Leptin resistance when associated visceral fat dysfunction, leads to pancreatic lipotoxicity followed by beta-cell apoptosis and diabetes onset, muscle insulin resistance, liver insulin resistance ${ }^{[18]}$.

ABSI was not found be statistically significant in patients with metabolic syndrome and diabetes mellitus. However ABSI was positively correlated with systolic BP and waist circumference in patients with metabolic syndrome. Our study was in accordance with prospective cohort study done Fujita et al who also concluded that ABSI was an inferior discriminator when compared to other anthropometric and lipid indices ${ }^{[19]}$. Sikandar Hayat Khan et al reported that ABSI is not a useful indicator in predicting metabolic syndrome and type 2 diabetes ${ }^{[20]}$. But our study was contradictory to a study done by which found ABSI levels to be statistically significant in patients with type 2 diabetes. They found that ABSI when used along with BMI is a better predicator of type 2 diabetes ${ }^{[21]}$.

LAP is an indicator that measure WC and estimate TG. LAP was found to be significantly increased in patients with metabolic syndrome and diabetes mellitus compared to controls in our study. LAP was found to be positively correlated with diastolic BP, systolic BP, triglycerides and WC in patients with type 2 diabetes mellitus and with triglycerides and waist circumference in patients with metabolic syndrome. Studies done by Khan ${ }^{[22]}$ and Xiang et al ${ }^{[23]}$ found that LAP is better biomarker than BMI for predicting metabolic syndrome and cardiovascular disorders. BMI reflects only excess weight when compared with LAP. Individuals with different metabolic risk profile may have a similar BMI [24]. WC cannot distinguish between subcutaneous and visceral adipose tissue. So, an 
increased WC cannot indicate high-risk visceral fat ${ }^{[8]}$.A work done by Henry reported that LAP is a better indicator than BMI and is far superior in identifying diabetes mellitus ${ }^{[25]}$.Studies done by Evan et al concluded that LAP is a useful biomarker for identifying individuals at risk of cardiovascular disorders among T2DM patients ${ }^{[26]}$. In a study done by Cheng $\mathrm{Y}$ et al showed that metabolic syndrome increases risk of cardiovascular disease, and the LAP could be used to recognize the metabolic syndrome even in people without fatty liver disease ${ }^{[27]}$. Chiang and Koo concluded that LAP was found to be an accurate tool for predicting the risk of metabolic syndrome in Taiwanese people and will help in a primary care centre to decide on which patients require further evaluation ${ }^{[28]}$.

Limitations of our study include a small sample size and we have included only a few lipid and anthropometric indices.

\section{Conclusion}

Our study concluded that lipid indices LAP, VAI are better than anthropometric indices like BMI, WC and ABSI in predicting metabolic syndrome and type 2 diabetes. Anthropometric indices when used should be correlated with metabolic variables and clinical symptoms.

\section{Acknowledgments}

We thank all the individuals for willingly participating in this study. We thank Mrs. Brilly M Rose, Biostatistician, Department of Community Medicine, Sree Narayana Institute of Medical Sciences for helping us with the statistical analysis. We acknowledge the support of the management of Father Muller's Medical College and Laboratory staff.

\section{Funding}

None

\section{Competing Interests}

None declared

\section{References}

1. Augusthy A, Jeppu AK, Sahu S, Jawalekar S, Marakala V, Iqbal S. A study of liver functions in metabolic syndrome and Type 2 diabetes mellitus. Int J Med Res Rev 2016;4(4):470475.

2. Yashavanth HS, Bharath MS. Comparison of body mass index and lipid accumulation product as a better indicator of metabolic syndrome. Int J Adv Med 2017;4:728-33.

3. Amato et al.: Cut-off points of the visceral adiposity index (VAI) identifying a visceral adipose dysfunction associated with cardiometabolic risk in a Caucasian Sicilian population. Lipids in Health and Disease. 2011;10:183.

4. Amato MC, Pizzolanti G, Torregrossa V, Misiano G, Milano S, et al. Visceral adiposity index (VAI) is predictive of an altered adipokine profile in patients with Type 2 Diabetes.2014;PLoS ONE 9: e91969.

5. Matthews D, Hosker J, Rudenski A, Naylor BA, Treacher $\mathrm{DF}$, et al.Homeostasis model assessment: insulin resistance and $\beta$-cell function from fasting plasma glucose and insulin concentrations in man. Diabetologia ,1985;28: 412-419.

6. Stefan N, Haring HU, Hu FB, Schulze MB.Metabolically healthy obesity: epidemiology, mechanisms, and clinical implications. Lancet Diabetes Endocrinol,2013; 1: 152-162.

7. Gomez-Peralta et al. Diabetol Metab Syndr 2018;10:21.

8. C Xia, R Li, S Zhang, L Gong, W Ren, Z Wang, Q Li. Lipid accumulation product is a powerful index for recognizing insulin resistance in non-diabetic individuals. European Journal of Clinical Nutrition 2012; 66: 1035-1038.

9. Huang PL. A comprehensive definition for metabolic syndrome. Dis Model Mech. 2009 May-Jun;2(5-6):231-7.

10. Amato MC, Giodano C, Galia M, Criscimanna A, Vitabile S, et al.Visceral Adiposity Index. Diabetes Care;2010 33: 920-922.

11. Krakauer NY, Krakauer JC. A new body shape index predicts mortality hazard independently of body mass index. PLoS ONE. 2012;7(7):e39504.

12. G. Bedogni, H. S. Kahn, S. Bellentani, and C. Tiribelli. A simple index of lipid overaccumulation is a good marker of liver steatosis. BMC Gastroenterology 2010;10(98).

13. Kirk, E. P., \& Klein, S. Pathogenesis and pathophysiology of the cardiometabolic syndrome. The Journal of Clinical Hypertension, 2009;11(12), 761-765.

14. Yan, L. L. et al. Midlife body mass index and hospitalization and mortality in older age.Jama.2006; 295, 190-198.

15. Zhang Y, Fu J, Yang S, Yang M, Liu A, et al. Prevalence of metabolically obese but normal weight (MONW) and metabolically healthy but obese (MHO) in Chinese Beijing urban subjects. Biosci Trends.2017; 11: 418-426.

16. Wu J, Gong L, Li Q, Hu J, Zhang S, Wang Y, Zhou H, Yang S \& Wang Z. A Novel Visceral Adiposity Index for Prediction of Type 2 Diabetes and Pre-diabetes in Chinese adults: A 5-year prospective study. Scientific Reports 7: 13784 .

17. Wang, Y. et al. Predictive value of visceral adiposity index for type 2 diabetes mellitus: A 15 -year prospective cohort study. Herz40,2015;(3), 277-281.

18. Unger RH, Scherer PE: Gluttony, sloth and the metabolic syndrome: a roadmap to lipotoxicity. Trends Endocrinol Metab 2010, 21:345-352.

19. Fujita M., Sato Y., Nagashima K., Takahashi S., Hata A. Predictive power of a body shape index for development of diabetes, hypertension, and dyslipidemia in Japanese adults: A retrospective cohort study. PLoS ONE. 2015;10:e0128972.

20. Khan SH, Shahid R, Fazal N, Ijaz A. Comparison of various abdominal obesity measures for predicting metabolicsyndrome, diabetes, nephropathy, dyslipidemia. J Coll Physicians Surg Pak 2019; 29(12):1159-1164. 
21. Bawadi H, Abouwatf M, Alsaeed S, Kerkadi A,Shi Z Body Shape Index Is a Stronger Predictor of Diabetes. Nutrients. 2019 ; 11(5): 1018.

22. Kahn, H. S. The" lipid accumulation product" performs better than the body mass index for recognizing cardiovascular risk: A population-based comparison. BMC Cardiovascular Disorders, 2005; 5(1), 26.

23. Xiang, S, Hua, F Chen, L Tang, Y Jiang, Liu, Z .Lipid accumulation product is related to metabolic syndrome in women with polycystic ovary syndrome. Experimental and Clinical Endocrinology \& Diabetes.2013;121(02), 115-118.

24. Despre JP, Lemieux I, Bergeron J, Pibarot P, Mathieu $\mathrm{P}$, Larose $\mathrm{E}$ et al. Abdominal obesity and the metabolic syndrome: contribution to global cardiometabolic risk. Arterioscler Thromb Vasc Biol 2008; 28: 1039-1049.
25. Khan HS. The Lipid Accumulation Product Is Better Than BMI for Identifying Diabetes. Diabetes care 2006;29(1).

26. Adu et al. Lipid accumulation product (LAP) index as a potential risk assessment for cardiovascular risk stratification among type II diabetes mellitus in a Ghanaian population: A cross-sectional study, Cogent Medicine (2019), 6: 1639880.

27. Cheng $Y$ et al. Fatty Liver Index and Lipid Accumulation Product Can Predict Metabolic Syndrome in Subjects without Fatty Liver Disease. Hindawi Gastroenterology Research and Practice Volume 2017, 9279836, 12 pages.

28. Chiang and Koo: Lipid accumulation product: a simple and accurate index for predicting metabolic syndrome in Taiwanese people aged 50 and over. BMC Cardiovascular Disorders 2012;12:78

*Corresponding author:

Dr. Asha Augusthy, Professor and HOD, Department of Biochemistry, Sree Narayana Institute of Medical Sciences, North Kuthiyathodu PO, Ernakulam, Kerala Phone: +919744111079

Email: asha.augusty@gmail.com

Date of Submission : 22/04/2021

Date of Final Revision : 01/06/2021

Financial or other Competing Interests: None.

Date of Acceptance : 05/06/2021

Date of Publication : 30/06/2021 